Revue

del'histoire des religions

\section{Revue de l'histoire des religions}

$4 \mid 2013$

Sermo mysticus Mystique et langage entre Moyen Âge et époque moderne

\title{
La quête du pur amour à travers la Loi d'amour ou Quatrième Abécédaire spirituel de Francisco de Osuna (1530)
}

The quest for pure love in the Law of Love or Fourth Spiritual Alphabet by

Francisco de Osuna (1530)

\section{Estelle Garbay-Velázquez}

\section{OpenEdition}

Journals

Édition électronique

URL : http://journals.openedition.org/rhr/8178

DOI : 10.4000/rhr.8178

ISSN : 2105-2573

Éditeur

Armand Colin

Édition imprimée

Date de publication : 1 décembre 2013

Pagination : 583-608

ISBN : 978-2200928667

ISSN : 0035-1423

\section{Référence électronique}

Estelle Garbay-Velázquez, « La quête du pur amour à travers la Loi d'amour ou Quatrième Abécédaire spirituel de Francisco de Osuna (1530) », Revue de l'histoire des religions [En ligne], 4 | 2013, mis en ligne le 01 décembre 2016, consulté le 20 avril 2019. URL : http://journals.openedition.org/rhr/8178 ; DOI $10.4000 /$ rhr.8178 


\section{La quête du pur amour à travers la Loi d'amour ou Quatrième Abécédaire spirituel de Francisco de Osuna (1530)}

Un siècle avant le Guide spirituel de Miguel de Molinos, la Loi d'amour ou Quatrième Abécédaire spirituel (1530) du franciscain andalou Francisco de Osuna est déjà traversé par une intense quête spirituelle de pur amour, dans un contexte marqué par la condamnation des illuminés de Tolède. À travers des images et des métaphores qu'il emprunte aux mystiques rhéno-flamands tels Henri Harphius, Francisco de Osuna construit en langue castillane un discours mystique qui prend par endroits la tournure d'un plaidoyer pour une quête spirituelle orthodoxe de pur amour, contre les erreurs et les excès des théories de l'Abandon. Pour ce faire, il tente d'asseoir sa doctrine du pur amour sur une base théologique et doctrinale solide, afin d'écarter tout soupçon d'hétérodoxie qui pesait sur les enseignements spirituels du Recueillement.

The quest for pure love in the Law of Love or Fourth Spiritual Alphabet by Francisco de Osuna (1530)

One century before the Spiritual Guide of Miguel de Molinos, the Law of Love or Fourth Spiritual Alphabet (1530) by the Andalusian Franciscan writer Francisco de Osuna already witnesses an intense spiritual quest for pure love, in the context of condemning Toledan Alumbrados ("enlightened ones"). Through images and metaphors recalling the Flemish mystical writings of Henry Herp, Francisco de Osuna constructs a mystical discourse in Castillian which sounds like a plea for an orthodox spiritual quest for pure love, against the errors and the excesses of the "Dejados" theories. To do so, he attempts to base his doctrine of pure love on a solid ground that is both theological and doctrinal, in order to brush aside any suspicion of heterodoxy that hung over the spiritual teachings of "Recollection".

Revue de l'histoire des religions, 230 - 4/2013, p. 583 à 608 
L'Espagne du début du XVI ${ }^{\mathrm{e}}$ siècle, marquée par l'empreinte du cardinal Cisneros, offre un riche éventail d'écrivains mystiques - parmi lesquels bon nombre de franciscains tels que Alonso de Madrid, Francisco de Osuna, Francisco Ortiz, Bernardino de Laredo ou Bernabé de Palma -, qui ont témoigné d'une vive et intense vie spirituelle marquée indéniablement par une quête incessante de pur amour. Parmi ces auteurs - que Jacques Le Brun passe sous silence dans son ouvrage consacré aux principales manifestations littéraires du pur amour avant et après Fénelon ${ }^{1}-$, Francisco de Osuna est très certainement un des premiers et des plus éminents écrivains mystiques à avoir sérieusement exposé et défendu la doctrine du pur amour en langue castillane. Il l'a fait en pleine controverse spirituelle, quand sont remis en cause puis condamnés les illuminés de Tolède, qui clamaient haut et fort que la perfection résidait en un total abandon à une forme vague de pur amour de Dieu. Sans attendre les querelles spirituelles et théologiques du XVII ${ }^{\mathrm{e}}$ siècle, et plus d'un siècle avant la rédaction et la mise à l'index du Guide spirituel de Miguel de Molinos, la doctrine du pur amour avait donc déjà suscité en Espagne un large débat. Et c'est dans ce cadre qu'était publiée, en 1530, une Quatrième partie de l'Abécédaire spirituel intitulée Loi d'amour ${ }^{2}$, alors que l'Abécédaire initialement conçu par Francisco

1. Jacques Le Brun, Le pur amour de Platon à Lacan, Paris, Seuil («La librairie du XXI ${ }^{\mathrm{e}}$ siècle»), 2002.

2. Étant donné qu'il n'existe aucune traduction française du Quatrième Abécédaire spirituel, pas plus que d'édition espagnole récente satisfaisante - l'édition de la Ley de amor y cuarta parte préparée par Juan Bautista Gomis dans Místicos franciscanos españoles, Madrid, Biblioteca de Autores Cristianos (BAC), 1948, tome 1, p. 217-700 ne constitue pas une référence fiable -, toutes nos références renverront à l'édition princeps de 1530 (Séville?), que nous avons transcrite et modernisée: voir Estelle Garbay-Velázquez, Étude et édition annotée $d u$ "Quatrième Abécédaire spirituel» de Francisco de Osuna (1530), thèse de doctorat (inédite), Université de Toulouse 2 Le Mirail, 2012, pour l'édition p. 207-708. En revanche il est désormais possible de lire les cinq autres tomes de l'Abécédaire d'Osuna dans des éditions espagnoles récentes et fiables: Primer abecedario espiritual, édition de José Juan Morcillo Pérez, Madrid, Librería Editorial Cisneros, 2004; Segundo abecedario espiritual, édition de José Juan Morcillo Pérez, Madrid, Librería Editorial Cisneros, 2004; Tercer abecedario espiritual, introduction et notes de Saturnino López Santidrián, Madrid, BAC ( «Místicos franciscanos españoles », 2) 1998; Abecedario espiritual, Vy VI partes, édition de Mariano Quirós García, Madrid, Universidad Pontificia de Salamanca (Fundación Universitaria Española), 2002. La seule traduction française - partielle 
de Osuna comme manuel spirituel destiné à guider le chrétien dans sa quête intérieure de recueillement ne devait comporter que trois volumes. Et il se pourrait bien que ce quatrième tome paru en $1530^{3}$, cinq ans après la condamnation officielle des illuminés de Castille par l'Inquisition de Tolède, et en pleine vague de procès, soit un plaidoyer pour une quête spirituelle «orthodoxe» du pur amour, qui semblait à l'époque gravement discréditée et compromise par les excès et les erreurs des dejados, partisans de l'Abandon ${ }^{4}$. On s'essaiera ici à donner un aperçu du discours spirituel et mystique $\mathrm{du}$ franciscain andalou, à travers notamment un éventail d'images et de métaphores qui font écho aux grands textes des mystiques rhéno-flamands tels que Jean de Ruysbroeck ou Henri Harphius. On examinera ensuite la teneur du discours théologique élaboré, dans un contexte polémique, par Osuna afin de légitimer et d'asseoir l'expérience mystique du pur amour sur une base doctrinale solide, bien distincte des théories de l'Abandon.

\section{LE PUR AMOUR, CLEF DE VOÛTE DE L'ÉDIFICE SPIRITUEL DE LA LOI D'AMOUR}

La Ley de amor y cuarta parte del Abecedario espiritual n'est pas, à proprement parler, une œuvre mystique, malgré ce que les spécialistes d'Osuna ont longtemps maintenu, dans la mesure où la recherche de l'union avec Dieu dans l'amour n'occupe qu'un

\footnotetext{
- d'une œuvre d'Osuna dont on dispose pour l'heure est celle de Michel Darbord: Le recueillement mystique. Troisième Abécédaire spirituel [extraits], Paris, Cerf («Sagesses Chrétiennes »), 1992.

3. Ley de amor y quarta parte del Abecedario espiritual: donde se tratan muy de rayz los misterios y preguntas y exercicios del amor: y la theologia que pertenesce no menos al entendimiento que a la voluntad: harto util aun para los predicadores que dessean ver en buen romance las cosas que de si son escabrosas, (Séville?), 1530.

4. Lorsque les mots Abandon et Recueillement renvoient à des tendances ou mouvements spirituels aux pratiques bien codifiées, au-delà du sens habituel que comportent ces mots dans la langue commune, ils seront toujours précédés d'une majuscule. L'Abandon désigne ainsi un nouveau courant de spiritualité castillan qui, dans les années 1520 , tend à faire école et prône un abandon radical à une forme de pur amour de Dieu. Quant au Recueillement, il s'agit d'une autre tendance répandue chez les franciscains de l'époque, qui se base sur une pratique spirituelle d'introspection et de recueillement, et dont Francisco de Osuna est l'un des premiers théoriciens.
} 
espace réduit dans le Quatrième Abécédaire. Il s'agit bien plutôt d'une somme de théologie franciscaine sur le thème de l'amour divin et de l'amour humain: l'auteur, en effet, a renoncé à structurer ses chapitres suivant l'ordre alphabétique qu'il s'était imposé dans les trois premiers tomes, pour revenir à un ordre logique des traités classiques, ne conservant d'abécédaire que le nom. Il y ambitionne dès le prologue d'embrasser l'ensemble de l'enseignement chrétien sur le thème de l'amour, depuis la Bible jusqu'aux grands théologiens franciscains médiévaux, en passant par les différentes gloses, les Pères grecs et latins, sans négliger pour autant les philosophes païens de l'Antiquité, et adopte pour ce faire une démarche de théologie positive, tout en reconnaissant à plusieurs endroits la supériorité et l'excellence de la démarche de la théologie négative d'un pseudo-Denys.

Si l'on considère l'ensemble de ce vaste traité méthodique et exhaustif sur l'amour de 211 folios, force est de constater que le concept de pur amour n'y occupe qu'une place très limitée. L'examen des titres de ses cinquante-et-un chapitres est, à cet égard, hautement révélateur: un seul contient une référence explicite au pur amour, à travers l'expression el apurado amor (chapitre 25). Et si le thème est certes abordé sporadiquement au cours des chapitres $22,25,26,32,35,42$ et 48 , il ne fait véritablement l'objet d'un développement approfondi qu'aux chapitres 22,25 et 48 .

Le chapitre 22, consacré aux varones fervientes, c'est-à-dire aux plus fervents amoureux de Dieu, introduit pour la première fois l'idée d'un amour «pur». Car si tout un chacun est tenu d'aimer Dieu «de tout son cœur, de toute son âme et de toutes ses forces » (Deutéronome, 6,5 ) en ces occasions précises où il se doit de remplir l'obligation contenue dans le grand commandement, les «justes», les «fervents», qui s'engagent dans la voie de la perfection, s'efforcent d'aimer Dieu « continûment», suivant en cela le Proverbe 5, 19 attribué à Salomon ${ }^{5}$. Plus encore, les fervents amoureux de Dieu aspirent à aimer Dieu «infiniment». Et comment en serait-il autrement? Dieu, qui est par essence «immense et infini», mérite qu' on l'aime le plus possible, le plus parfaitement possible et, pour ainsi dire, «infiniment». Osuna

5. «Cerva charissima, et gratissimus hinnulus: / Ubera eius inebrient te in omni tempore, / In amore eius delectare iugiter», qu'Osuna traduit de la façon suivante: «Deléitate continamente en el amor de él». 
cite et traduit cette fois-ci un commentaire de Richard de SaintVictor sur le Cantique des cantiques $(1,3)$ : «Et en ce sens, je pense qu'il a été dit dans le Cantique des Cantiques "les jeunes filles t'ont beaucoup aimé", car Dieu, à qui va leur amour, est immense et infini ; c'est pourquoi l'amour n'aura ni fin ni mesure $»^{6}$.

Et même si Osuna ne manque pas de préciser que l'amour infini n'est pas de ce monde, les fervents amoureux, selon lui, possèdent en eux un désir infini de Dieu, qui est en quelque sorte un prélude et une préparation au plein et parfait amour qu'il leur sera effectivement donné de goûter dans l'état de béatitude. Du concept d'infinitude à celui de pureté, il n'y a qu'un pas. Pour Osuna, c'est en effet une propriété intrinsèque de l'amour pour Dieu que de tendre à la pureté, à l'inverse de l'amour terrestre, qui se mêle sans cesse de «vanités» et se change progressivement en «scorie ${ }^{7}$.

Dieu attend donc des hommes - et à plus forte raison des plus parfaits -, qu'ils l'aiment d'un amour pur, voire «purissime» (purísimo); il leur incombe d'y tendre de tout leur être et de prendre peu à peu conscience de la pureté de leur sentiment, tout en sachant qu'il demeure impossible à l'âme de connaître ici-bas le degré de pureté de l'amour qu'elle porte à Dieu :

Et les hommes fervents s'appliquent à ressentir et à connaître en eux-mêmes cet amour, et à le maintenir plus sincèrement et plus profondément que toute autre chose, à tel point que de fait ils connaissent mieux l'amour qu'ils éprouvent pour Dieu que leur propre respiration ou la vie elle-même. Et ne va pas penser pour autant qu'ils sont fiers d'eux-mêmes et tiennent en haute estime leur amour de Dieu, car si d'un côté ils sont conscients de cet amour, d'un autre côté ils doutent de sa pureté, notre Seigneur exigeant un amour pur, et même purissime ${ }^{8}$.

6. Ley de amor, ch. 22, f. 90v: «Y en este sentido pienso yo que fue dicho en los Cánticos "las doncellas te amaron mucho", porque infinito y inmenso es Dios, al cual va el amor de ellas; y, por tanto, no debe tener fin ni medida el amor ». Osuna traduit le latin de Richard de Saint-Victor: «Quo sensu et ergo dictum puto, Adolescentulae dilexerunt te nimis (Cant I). Infinitus quippe, et immensus est Deus in quem tendit amor [Col.1200A] earum, ut nullus esse debeat finis, vel mensura amoris» (Tractatus de gradibus charitatis, ch. II ; «Patrologia latina», Paris, 1855, vol. 196, col. 1199-1200).

7. Ley de amor, ch. 22, f. 93r: «Empero, el amor de Dios, así como es contrario al amor mundano, así tiene contrarias condiciones. El amor mundano se va cada día mezclando con más vanidades y se va tornando escoria; empero, el amor de nuestro Señor se afina más cada día y se hace más puro ».

8. Ibid: «Y los varones fervientes se trabajan por sentir y conocer en sí este amor, y continuarlo más y más verdadera y entrañablemente que otra cosa ninguna, 
Ainsi, en quelques mots, Osuna légitime la quête du pur amour, tout en la réservant aux plus parfaits: il leur appartient de prendre la mesure de la pureté de l'amour qu'ils éprouvent envers Dieu, «sans mélange d'aucune autre chose», de «vaquer à Dieu seul» et de «goûter secrètement son amour» dans le tréfonds de leur cœur et sans aucun intermédiaire?

Les jalons de la quête spirituelle du pur amour ainsi posés, Osuna s'adresse plus particulièrement, dans le chapitre 25 , à ceux qui se distinguent par le degré de perfection de leur cheminement spirituel, et qu'il nomme volontiers les «fervents et purs amoureux de Dieu» (el ferviente amador y puro de Dios): l'adjectif «pur» ne qualifie donc plus seulement l'amour pour Dieu, mais également celui qui se lance corps et âme dans une quête de pureté spirituelle à travers le divin amour, se retrouvant par là même hissé à une catégorie supérieure d'homme spirituel. Ce chapitre se propose, dès son titre: De algunos de los efectos que la ley del apurado amor hace en el ánima del varón perfecto («De quelques effets produits par la loi de l'amour épuré sur l'âme du parfait»), d'analyser quelques-uns des effets de «l'amour épuré» (el apurado amor) chez celui qui en fait l'expérience habituelle: ferveur contre toute forme de tiédeur spirituelle $(\text { tibieza })^{10}$, force croissante et constance (fortaleza) pour surmonter les obstacles et lutter contre les tribulations, indifférence

en tal manera que conocen más de hecho el amor que tienen a Dios cuando lo están amando que el resollar ni la vida; y, por esto, no pienses que están engreídos sobre sí, pensando que hacen mucho en amar a su Dios, porque, conociendo por una parte que aman a Dios, dudan por otra parte si está puro aqueste amor, ca nuestro Señor lo quiere puro y aun purísimo».

9. Soucieux d'éviter toute confusion théologique, Francisco de Osuna a pris soin de préciser (Ley de amor, ch. 22, f. 92v): «no por esto se sigue que no puedas tú conocer el amor que tienes a Dios en su pureza, como lo produces del corazón sin mezcla de otra cosa alguna, sino que sólo el amor sea medianero y solicitador entre el ánima y Dios cuando ella quiere vacar a él solo y gozar de él secretamente, sin que otro alguno entienda en ello, sino el solo amor entrañal y la bienquerencia del corazón. Y no pienses que por esto quiero decir que se pueda sentir el amor infuso, que agora no hablo sino del amor adquirido y producido de tu corazón para amar estudiosamente y con afeción a tu Dios». Lorsqu'il évoque le pur amour auquel aspirent les spirituels fervents, il est donc bien question d'amour «acquis », c'est-à-dire d'amour actif produit par la volonté humaine, et en aucun cas d'amour «infus », qui ne peut émaner que de la seule volonté divine.

10. Ibid., ch. 25, f. 101v: «El amor puro de Dios toda tribulación sufre, y no sería bueno si contino no creciese; y si alguna cosa lo atibia, no debe ser verdadero, ca, si lo fuese, cada día tomaría nuevas y mayores fuerzas ». 
aux louanges et aux injures de ce monde, absence de vanité (vanagloria $)^{11}$, etc.

Lorsque Osuna y exhorte ceux qui aspirent au pur amour enflammé (inflamado amor de Dios), à résister en toute intégrité aux remous et aux écueils de la mer du monde (las ondas del mar mundano), il pense certainement à ces illuminés (alumbrados) des années 1530, qui finirent par se retrouver, pour certains, sur les bancs du tribunal de l'Inquisition de Tolède: tel fut le cas des partisans de l'Abandon (dejados) - indirectement réprimandés par l'auteur de la Loi d'amour -, mais aussi de certains franciscains partisans du Recueillement, tel Francisco Ortiz, compagnon de route d'Osuna et ardent défenseur du pur amour devant le Saint-Office ${ }^{12}$.

Quant au chapitre 48 , situé quasiment à la fin de l'ouvrage et intitulé De los grados que tienen los que guardan la ley de amor («Les degrés par lesquels passent ceux qui observent la loi d'amour»), il fait la synthèse des différentes étapes de progression spirituelle en parcourant chacun des neuf degrés de l'échelle de la charité. Héritier d'une longue tradition d'auteurs ascétiques et mystiques qui ont disserté sur les différents degrés de l'amour et de la progression spirituelle à partir de l'image de l'échelle de Jacob (Genèse, 28, 10-22), Osuna rend un hommage particulier à Richard de Saint-Victor et à son Tractatus de gradibus charitatis. Mais à la différence de celui-ci, qui distingue quatre degrés dans la «violente charité», le franciscain andalou opte pour une division ternaire hautement symbolique. Il se fonde en premier lieu sur les trois étapes canoniques de la progression spirituelle décrites par Thomas d'Aquin - commençants (comenzantes, en latin

11. Ibid., ch. 25, f. 102v: «i Oh si sintiésemos cuánta fuerza tiene el puro amor de Dios, y cuán poderosa y generosa hace el ánima que lo posee ! ; Oh, cuán fuera irá de la tal ánima toda vanagloria y toda cosa que Dios no es ! ; Oh, cuán poco hemos gustado este amor cuando echamos de ver que hay deshonras o honras! ; Oh, cuán poco teme las unas y ama las otras el ferviente amador y puro de Dios!»

12. Voir le procès contre Francisco Ortiz et en particulier sa Lettre à l'Amiral de Castille, retranscrite par Ángela Selke (El Santo Oficio de la Inquisición. Proceso de Fr. Francisco Ortiz (1529-1532), prologue de José Luis L. Aranguren, Madrid, Ediciones Guadarrama, 1968, annexe V, p. 388): «Mas sobre todo será bienaventurado el que se despertare a servir a Dios por consideración de la infinita bondad de Dios que en sí tiene, por la qual sin otro respecto merece que toda criatura le sirva y le ame, y más vale una centella deste puro amor que mucho amor interesal». 
incipientes), progressants (aprovechantes, en latin proficientes) et parfaits (perfectos, du latin perfecti) -, qui équivalent aux trois catégories de la perfection angélique selon le pseudo-Denys: purificatio, illuminatio et perfectio. Purification, illumination et perfection, voilà pour Osuna, dans la droite ligne de la triple voie bonaventurienne, quelles sont les trois grandes étapes de la progression spirituelle, qui peuvent se subdiviser chacune à leur tour en trois niveaux, aboutissant ainsi aux neuf degrés de la charité, l'amour pouvant seul permettre à l'homme de gravir les échelons qui vont au ciel.

Il convient de faire remarquer que si les neuf degrés de l'amour correspondent en nombre avec ceux mis en évidence par le mystique flamand Henri Harphius dans son ouvrage Miroir de perfection ${ }^{13}$, le contenu qualitatif des degrés de l'amour varie sensiblement d'un auteur à l'autre. Francisco de Osuna, pour rendre son propos moins abstrait, a recours à l'image du temple de Salomon, dont la structure tripartite du plan figure les trois étapes de la vie spirituelle: la partie extérieure à ciel ouvert (les trois cours) représente l'étape de purification qui incombe aux commençants; l'espace intérieur sacré réservé aux prêtres symbolise la phase d'illumination propre aux progressants; tandis que l'espace secret très sacré accessible au seul grand prêtre fait figure de la perfection amoureuse restreinte aux plus parfaits. Et de même que les trois Hiérarchies célestes se décomposent chacune en trois ordres d'anges, les trois parties du Temple de Salomon se divisent à leur tour en trois sous parties bien distinctes, qui figurent chacun des neuf degrés de l'amour de Dieu, suivant le schéma suivant:

$1^{\text {er }}$ degré - Les débutants ou apprentis 1 : ceux qui s'appliquent à l'amour de Dieu à travers la pratique de certaines œuvres extérieures, mais qui n'ont pas encore quitté l'état de péché.

$2^{e}$ degré-Les débutants ou apprentis 2 : ceux qui s'appliquent à l'amour de Dieu, par exemple à travers la prière, et qui sont sortis délibérément de l'état de péché, mais qui y retombent encore parfois.

13. Le titre original de cette œuvre publiée tout d'abord en flamand en 1475 est Spieghel der Volkomenheit, avant d'être traduite en latin comme Directorium aureum contemplativorum (1509). En 1538 elle paraît sous le titre de Theologia mystica cum speculativa. 
$3^{e}$ degré - Les débutants ou apprentis 3 : ceux qui pratiquent assidûment les pénitences corporelles pour ne plus retomber dans le péché.

$4^{e}$ degré - Ceux qui progressent 1 : ceux qui pratiquent la pénitence spirituelle et s'initient aux matières spirituelles.

$5^{e}$ degré - Ceux qui progressent 2: ceux qui découvrent le goût des matières spirituelles (qui n'est donné qu'à ceux qui ont déjà une grande pratique et une compréhension des exercices spirituels).

$6^{e}$ degré - Ceux qui progressent 3: ceux qui s'élèvent vers la pleine connaissance d'eux-mêmes.

$7^{e}$ degré - Les parfaits 1 : ceux qui s'élèvent vers la connaissance de Dieu au moyen de la méditation continue des propriétés divines.

$8^{e}$ degré - Les parfaits 2: ceux qui pratiquent la contemplation (qui est réservée au cercle très étroit des plus familiers amis de Dieu et des choses secrètes).

$9^{e}$ degré - Les parfaits 3 : ceux qui s'adonnent au pur amour de Dieu, qui abandonnent tout autre exercice pour se contenter de la seule présence divine.

Le pur amour constitue donc le neuvième et dernier échelon de la progression spirituelle. Il est symbolisé par le propitiatoire situé au cœur du Temple de Salomon, la fameuse tabla de oro posée au-dessus de l'Arche de l'Alliance:

La dernière chose qui se trouvait dans le temple, autour de laquelle s'organisait tout le reste, était une table en or sans aucune peinture [...] Cette table, en or le plus pur, figure le plus pur amour de Dieu, qui appartient au plus haut degré des plus parfaits ${ }^{14}$.

Espace sacré accessible aux seuls initiés, le pur amour est en même temps le but ultime, la raison d'être qui donne tout son sens au parcours spirituel, et le trésor caché, la récompense que seuls goûteront les plus parfaits d'entre les parfaits.

À travers l'évocation des neuf degrés successifs de la charité, le franciscain veut montrer que l'itinéraire spirituel de l'âme vers Dieu est un parcours parfaitement balisé. Il met ainsi en garde les amoureux zélés qui voudraient brûler les étapes en se livrant dès le départ à une forme d'abandon mystique inconditionné, et

14. Ley de amor, ch. 48, f. 192r: «Lo último que estaba en el templo, a que todo lo otro se ordenaba, era una tabla de oro sin pintura alguna (...). Esta tabla, que era de oro purísimo, tiene figura del purísimo amor de Dios, que pertenece al último grado de los varones perfectos». 
qui prétendraient détenir une fois pour toutes la clé du pur amour en faisant fi de toute une phase d'initiation et d'ascèse pourtant nécessaire. En matière d'amour divin, on ne peut guère s'improviser «parfait»:

et ce n'est que très tard, dans sa vieillesse, qu'il [notre Seigneur] donna à Abraham le fils qu'il lui avait promis, pour nous montrer en cela que personne, dès les premiers pas, ne peut prétendre être parfait en amour saint, sans passer d'abord par l'amour des commençants, puis l'amour des progressants, pour arriver à l'amour des parfaits ${ }^{15}$.

D'autant plus que la perfection n'est pas de ce monde: l'ultime degré de l'amour ne sera jamais atteint une fois pour toutes icibas. Voilà pourquoi, explique Osuna ${ }^{16}$, il n'est pas précisé dans le Livre le nombre d'échelons, peut-être infini, que compte l'échelle de Jacob. Peu importe donc le nombre exact de degrés (le chiffre neuf exprime avant tout le nombre élevé et la complexité des étapes à franchir avant de pouvoir parvenir au terme de l'itinéraire): l'on retiendra surtout que le pur amour de Dieu constitue l'ultime degré de l'échelle de l'amour, la cime du mont de la perfection, la clef de voûte de tout l'édifice spirituel décrit dans la Loi d'amour: il est la raison d'être et l'aboutissement du voyage, le principe qui meut et oriente les pas du pèlerin amoureux de Dieu.

\section{IMAGES ET SYMBOLES DU PUR AMOUR DANS LA LOI D'AMOUR}

Il n'est pas inintéressant d'analyser la forme que prend, sous la plume d'Osuna, le discours mystique sur le pur amour à travers non seulement ses préférences lexicales, mais aussi les images et métaphores qui donnent corps et vie à son propos. Le défi de toute écriture mystique consiste à surmonter le problème de l'ineffabilité de l'expérience du divin amour, qui résiste à toute tentative de communication et de traduction. Comment dire l'indicible, appréhender l'impensable? Les Abécédaires spirituels du Franciscain andalou sont tout entiers traversés par ces inquiétudes et ces

15. Ibid., ch. 48, f. 189r-v: «y muy tarde en su vejez dio [nuestro Señor] a Abrahán el hijo que le había prometido, para nos enseñar en esto que ninguno es al primer vuelo perfecto en su sancto amor, sin que pasen por el amor de los que comienzan, y por el amor de los que aprovechan, y lleguen al amor de los perfectos».

16. Ibid. 
questionnements, et en particulier la Loi d'amour, dont le prologue s'ouvre sur un très beau paragraphe exprimant l'ineffabilité des mystères de l'amour:

Pour traiter comme il se doit des mystères de l'amour et que le style soit à la hauteur de leurs mérites, il me semble que la plume devrait être en or incandescent $[\ldots]$; l'encre pour l'écrire ne devrait pas être ordinaire, mais faite d'une liqueur ardente [...]. Les doigts aptes à tenir cette plume légère ne devraient pas être ceux d'un homme pur, mais ceux du Dieu vivant ${ }^{17}$.

Et parmi ces mystères, y a-t-il matière plus subtile et ineffable que le «pur amour»? Au chapitre 48, Osuna souligne à plusieurs reprises la difficulté de $«$ donner clairement à sentir $»^{18}$ ce point délicat. Pour y remédier, il a donc recours à des images et métaphores qui se superposent et s'entremêlent. Et si la plume du mystique doit être «d'or» et «de feu», c'est sans doute pour tenter d'égaler les vertus du pur amour, qui sont comparables en premier lieu à celles de l'or et du feu.

Lorsque Osuna choisit de figurer en priorité le pur amour par le propitiatoire du Temple de Salomon, il fonde sa comparaison en premier lieu sur la noblesse et le caractère précieux de la matière de cette table sacrée - l'or-, mais surtout sur sa pureté. La comparaison se révèle particulièrement féconde sous la plume d'Osuna, qui peut ainsi donner à sentir concrètement, avec la plasticité d'images évocatrices, le processus de purification du sentiment amoureux, qui courrait sans cela le risque de demeurer une abstraction stérile et absconse. En choisissant de qualifier le pur amour non plus par l'adjectif puro mais par le participe passé apurado, il renvoie directement au champ lexical de l'affinage des métaux précieux, et en particulier celui de l'or ${ }^{19}$. De même que ce dernier, pour devenir pur, doit être tout d'abord épuré et systématiquement nettoyé de

17. Ley de amor, prologue, f. $4 \mathrm{v}$ : «Para que fuesen dignamente escritos los misterios del amor y el estilo de decir igualase con su alto merecimiento, paréceme que la péndola había de ser de oro hecho fuego (...); la tinta de esta escritura no había de ser común, ca se debería escrebir con mosto ferviente (...). Los dedos que trajesen esta ligera pluma no habían de ser de hombre puro, sino de Dios vivo ».

18. «No se puede dar muy claramente a sentir este punto [...]», Ley de amor, ch. 48 , f. $192 \mathrm{v}$.

19. Le verbe apurar, qui traduit le latin purgare, est défini par le Diccionario de Autoridades comme l'action d'affiner, de purifier les métaux tels l'or ou l'argent en les nettoyant, en leur ôtant toute impureté, tout résidu de corps étranger au moyen d'un creuset: «Purificar y limpiar de excrementos y de la materia crassa 
tout résidu de corps étranger, l'amour de Dieu ne sera vraiment pur qu'après avoir subi un processus similaire de purification: si l'amour veut approcher la pureté de l'or, il conviendra de se débarrasser de toute autre considération et de fixer son attention sur Dieu seul, sans mélange d'aucune autre affection. Au chapitre 48, il tire habilement parti de la métaphore au moyen d'une opposition efficace, afin de traduire la supériorité du pur amour (or pur) sur les bonnes œuvres (métaux dorés):

De même que l'or donne éclat, beauté et valeur à tous les autres métaux, de même l'amour rend agréables toutes les œuvres et services divins. Cependant, il y a une différence entre l'amour lui-même et les œuvres d'amour, tout comme entre l'or et les ouvrages dorés. Tous ceux qui se consacrent à la pratique des bonnes œuvres le font par amour et avec le plus grand soin pour plaire à Dieu; néanmoins, ceux qui se consacrent exclusivement à l'amour, une fois qu'ils ont accompli en temps voulu avec amour leurs obligations, vaquent tout le reste du temps au seul amour. La différence est grande entre dorer et forger l'or pur. L'argent doré est très précieux, mais l'or infiniment pur du propitiatoire surpasse tout. Il semble insensé que dans le domaine de l'amour il n'existe pas d'œuvre qui se suffise à elle-même, alors qu'il en existe dans tous les autres domaines de ce monde. L'or infiniment pur est par lui seul source de contentement, et celui qui se consacre au seul amour fait un propitiatoire d'or infiniment pur ${ }^{20}$.

Il est certes tout à fait licite et nécessaire de pratiquer la charité et autres bonnes œuvres, mais ces dernières ne sont que du métal doré au regard du pur amour, sentiment divin par excellence qui se suffit à lui-même et qui surpasse toute autre occupation. À côté de ceux qui se contentent de pratiquer les bonnes œuvres, et qui sont comparables à des doreurs d'argent, Osuna fait des parfaits en quête de pur amour des orfèvres qui travaillent l'or le plus pur.

alguna cosa, como la plata, oro y otros metales, acrisolándolos y purgándolos de las heces que tienen para que queden acendrados».

20. Ley de amor, ch. 48, f. 192r: «así como el oro da lustre y hermosura y valor a todos los otros metales, así el amor hace agradables todas las obras y servicios de Dios. Empero, diferencia hay del mesmo amor a las obras del amor, así como la hay entre el oro y las obras doradas. Todos los que se ejercitan en buenas obras tiénenlas por el amor muy esmeradas para agradar a Dios, mas los que en sólo el amor se ejercitan hacen con amor a sus tiempos lo que son obligados, y todo lo demás vacan al solo amor. Mucho va de dorar a labrar en puro oro. Muy buena es la plata dorada, mas el oro purísimo del propiciatorio es mejor que todas las otras cosas. Cosa parece ajena de razón que no tenga el amor obra por sí que a sólo él convenga, pues la tienen todos los otros negocios del mundo. Por sí solo aplace el oro purísimo, y el que en sólo amor se ejercita hace propiciatorio de oro purísimo ». 
Mais cet or n'atteindrait jamais la pureté sans l'action purificatrice du feu, deuxième élément symbolisant le pur amour chez Osuna. Les images ignées sont récurrentes dans la Loi d'amour. Et si la tiédeur (tibieza) vient à signifier le manque d'amour de Dieu et le désamour en général, la chaleur ardente du feu, la brûlure et les images incandescentes renvoient bien souvent à la consumation du pur amour en Dieu (ainsi la métaphore du «four perdurable d'amour de Dieu $\left.{ }^{21} \gg\right)$. Le franciscain souligne dès le chapitre 22 les propriétés purificatrices et régénératrices du «feu du divin amour», sans commune mesure avec les propriétés destructrices du feu terrestre ${ }^{22}$. Et de la même façon que l'on pouvait distinguer différents degrés de pureté dans l'or, le feu peut se révéler plus ou moins pur, suivant qu'il brûle de lui-même, ou qu'il a besoin d'être alimenté par du bois ou de la poudre ${ }^{23}$.

Ainsi l'amour sera d'autant plus pur et plus parfait qu'il trouvera son plein contentement dans l'être aimé - Dieu -, sans qu'il soit nécessaire de l'attiser par d'autres exercices.

À côté du feu, et aussi paradoxal que cela puisse paraître, l'élément liquide sert, plus rarement, à Osuna pour évoquer d'autres caractéristiques du pur amour. Chez notre auteur, d'une façon générale, l'eau (el agua) symbolise plutôt ce qui est le plus étranger à l'amour (la mer de l'amour du monde, les tribulations, la froideur du désamour...) et qui vient «éteindre, «gâcher» ou «diluer» la pureté de l'amour. C'est bien ce qu'exprime le verbe aguar, que le franciscain utilise lorsqu'il entend signifier que l'intérêt inhérent à l'amour humain peut dans certains cas altérer, voire amoindrir la pureté de l'amour de Dieu ${ }^{24}$.

Mais lorsque l'élément liquide entre en contact avec l'élément igné et se réchauffe, il devient à son tour porteur d'images évocatrices de l'expérience mystique de l'amour de Dieu dans toute son intensité: celui qui parvient à éprouver un amour pur qui soit tout entier tourné vers le seul Dieu, sans mélange d'aucune autre chose, expérimente

21. Ibid., ch. 22, f. 93v : «el horno perdurable del amor [de Dios]».

22. Ibid., f. $94 \mathrm{v}$.

23. Ley de amor, ch. 48, f. 192r-v: «Más excelente es el fuego que arde en sí mesmo que no el que arde en leña o en pólvora o en otra cosa alguna; y así, es más excelente el amor que vive en sí solo que no el que ha menester leña de otros algunos ejercicios para despertarse y recrearse: entonces es purísimo y perfecto el amor, cuando sabe vivir por sí solo y no ha menester sino al amado».

24. Ibid., ch. 42, f. 170v (texte cité infra note 37). 
une sensation de vive brûlure suivie d'un «bouillonnement» de ses entrailles, comme si celles-ci passaient, sous l'effet du feu de l'amour divin, de l'état liquide à l'état gazeux, et s'élevaient alors merveilleusement vers Dieu, se mêlant à l'éther: «Lorsque chez les parfaits règne l'amour sans partage, les entrailles bouillonnent et brûlent, les affections se fortifient, le cœur s'embrase, et les sens s'éveillent à une chose d'autant moins explicable qu'on la ressent plus intensément $»^{25}$.

Par ailleurs, l'eau peut aussi, sous la plume du franciscain, évoquer le pur amour à travers l'image de la source (la fuente), symbole par excellence de pureté, à laquelle l'âme du parfait, emplie d'amour de Dieu, s'abreuve telle une «éponge»: alors l'âme «se rend compte que tout ce qu'elle faisait jusqu' alors n'était que fiction, convaincue que tout est mensonge en dehors de l'amour ${ }^{26}$.

Enfin, il ne faudrait pas oublier, parmi les symboles du pur amour, le sel (la sal). De la même façon que ce dernier relève le goût de tous les aliments, l'amour de Dieu est seul apte à donner de la saveur aux autres exercices spirituels et à toute occupation quelle qu'elle soit, les rendant paradoxalement «doux» (dulces):

Aimer plaît à tel point à ceux qui y goûtent qu'ils ne trouvent plus de goût à rien d'autre; aimer, pensent-ils, est le sel de tous les autres exercices, et lorsque, en bonnes brebis du Seigneur, ils se nourrissent de ce seul sel, ils se contentent au plus haut point, et découvrent que rien n'est doux comme l'amour, qui rend douce toute chose ${ }^{27}$.

L'on remarquera que les métaphores du pur amour présentes dans la Loi d'amour renvoient toutes à des éléments naturels (le feu, l'eau, et indirectement l'air) ou à des matériaux considérés comme précieux (l'or et le sel). Il s'agit dans presque tous les cas de matière à l'état brut, certes susceptible de subir un processus de purification, mais étrangère à toute mise en forme ou fabrication artisanale. $\mathrm{Ne}$

25. Ley de amor, ch. 48, f. 192v: «Cuando reina sólo el amor en los varones perfectos, bullen y hierven las entrañas, y fortalécense las afeciones, y enciéndese el corazón, y avívase el sentido a una cosa que tanto menos se puede explicar cuanto más se siente».

26. Ibid.: «Está el ánima como esponja que ha dado consigo en la fuente del amor, y hace cuenta que todo lo que hasta entonces hacía era fingimiento, creyendo que todo es burla sino amar».

27. Ibid. : «Agrada en tanta manera el amar a los que lo saben que en ninguna otra cosa sino en él toman sabor; a él tienen por sal de todos los otros ejercicios, y, cuando como buenas ovejas del Señor comen sola esta sal, se hallan más contentos, y conocen que sólo el amor que hace dulce todo lo demás es más dulce». 
fait exception que le propitiatoire du Temple, «une table rase en or infiniment pur», mais Osuna prend soin de préciser «sans aucune peinture»: car, écrit-il, «c'est bien plus grande perfection pour l'homme que d'éprouver un amour en soi si pur qu'il n'y associe aucune figure de créature, [et] c'est cet amour qui est figuré par le propitiatoire $»^{28}$.

Osuna veut faire comprendre par là que l'expérience mystique du pur amour échappe à toute mise en forme: ses contours se dérobent et elle demeure au-delà de toute représentation. La spiritualité du pur amour est volontiers iconoclaste: elle est une quête incessante d'intériorité, de simplicité, d'authenticité. Loin des apparences, des rites, et des ornements, seul le sentiment d'amour de Dieu importe, dans toute sa pureté.

Ces images n'ont en elles-mêmes rien de très novateur: les quatre champs lexicaux hautement symboliques sont omniprésents dans le Livre - auquel le franciscain s'est abreuvé -, avant d'être inlassablement repris dans les gloses successives des Pères et Docteurs de l'Église, de sorte qu'il serait vain de tenter ne serait-ce que l'ébauche d'un inventaire du corpus textuel source. L'on remarquera cependant que toutes ces images - or, feu, bouillonnement, effervescence et sel - se trouvent précisément réunies pour évoquer, en des termes très similaires, l'expérience du pur amour dans les écrits mystiques d'Henri Harphius. Teodoro Martín $^{29}$ a déjà signalé le Directorium comme étant une source textuelle privilégiée, quoique non avouée, du franciscain d'Osuna. Qu'il y ait eu une influence réelle de ce texte - les premières éditions du Directorium en latin (Cologne, 1509 et 1513; Anvers, 1516) circulèrent très tôt en péninsule Ibérique - sur la prose du Quatrième Abécédaire, est indéniable: il reste encore à en définir les contours et les modalités.

28. Ibid.: «muy más perfecta cosa es tener hombre el amor por sí muy purísimo sin pintar en él cosa criada, porque tal como éste es el que se figura en el propiciatorio, que era una tabla rasa de oro purísimo que ninguna cosa tenía pintada en sí».

29. Voir la démonstration de Teodoro Martín Hernández dans son ouvrage Enrique Herp (Harphius) en las letras españolas, Talleres de El Diario de Ávila, Ávila, 1973, p. 50-64. Voir aussi son édition du Directorio de contemplativos, Madrid, Biblioteca de Autores Cristianos (BAC), 2004. 


\section{LA LOI D'AMOUR, UN DISCOURS THÉOLOGIQUE VISANT À LÉGITIMER L'EXPÉRIENCE MYSTIQUE DU PUR AMOUR, EN PLEINE POLÉMIQUE AUTOUR DE L'ABANDON}

Francisco de Osuna constitue un maillon important dans la chaîne des auteurs mystiques du pur amour: il emprunte aux mystiques rhéno-flamands tout un patrimoine lexical et sémantique, qu'il est un des premiers à traduire du latin au castillan, reprenant, renouvelant et enrichissant ainsi une série de symboles déjà canoniques. Il convient de rappeler à cet endroit que le franciscain andalou est, dans les années 1520, un partisan convaincu de la vulgarisation des matières spirituelles, y compris mystiques. $\mathrm{Ne}$ met-il pas en effet à la portée de tous - et non plus seulement à la disposition des seuls religieux et aux théologiens versés en latin - sa méthode contemplative du Recueillement, exposée avec pédagogie dans le troisième tome de son Abécédaire spirituel (1527), livre de chevet de Thérèse d'Avila? Exprimer en langue vernaculaire les plus profondes et subtiles matières mystiques constituait un défi ambitieux et très délicat, avec le risque d'être mal compris ou mal interprété par des personnes sans formation théologique: la frontière entre la mystique orthodoxe et l'hérésie paraît bien souvent très fine. Or cette frontière avait, selon le chapitre des franciscains de Castille, déjà été franchie en 1524 par ceux que l'on appelait les dejados, défenseurs d'un radical abandon à une forme de pur amour de Dieu, qui aurait eu la propriété de rendre parfait, voire «impeccable», celui qui s'y serait livré, abstraction faite de toute autre considération, et à la marge des pratiques cultuelles fixées par l'institution ecclésiastique. L'Inquisition de Tolède s'était empressée de mettre officiellement à l'index cette nouvelle hérésie potentiellement subversive en publiant dès 1525 l'Édit de Tolède: celui-ci censurait 48 propositions attribuées aux «illuminés, abandonnés ou parfaits», et inaugurait une vague d'arrestations et de procès qui allait durer une dizaine d'années, impliquant certains frères franciscains. Voilà que la doctrine du pur amour était sérieusement compromise par les excès de la doctrine de l'Abandon, mettant du même coup en péril une partie de l'enseignement spirituel d'Osuna. Il devenait par conséquent urgent de redéfinir et de recadrer la quête spirituelle du pur amour dans les limites de la droite orthodoxie. Et c'est là à notre avis la raison 
d'être de cette Quatrième partie de l'Abécédaire spirituel ou Loi d'amour, qui, on l'a vu, n'était pas prévue initialement dans le plan de rédaction du grand Abécédaire osunien, conçu en trois tomes ${ }^{30}$.

Qu'elles soient dues à l'initiative de Francisco de Osuna ou que celui-ci se soit vu instamment prié de s'exécuter par les supérieurs de son Ordre, la rédaction et la publication en 1530 de la Loi d'amour avaient un enjeu double: légitimer et asseoir la quête de pur amour sur un important corpus d'autorités solidement établi, tout en réfutant les erreurs et en combattant les dérives de la théorie de l'Abandon. Voyons quelques exemples de réajustements doctrinaux $^{31} \mathrm{du}$ franciscain autour de la question du pur amour.

\section{Un amour pur et désintéressé :}

\section{la place de l'amour de soi et de l'amour du prochain}

À la différence des partisans de l'Abandon, il n'y a pas chez Osuna d'exigence de morale parfaitement désintéressée. Les procès contre les illuminés témoignent d'une aversion prononcée des dejados contre ce qu'ils nomment les propiedades, autrement dit contre ce qui provient de la propre initiative, ou contre ce qui pourrait cacher un intérêt personnel. Et ce, précisément, au nom du pur amour: l'amour de Dieu n'est vraiment pur que s'il est libre de toute inclination naturelle, de tout intérêt, et de tout ce qui émane en général de la volonté humaine. Celle-ci doit s'effacer, se suspendre et se conformer en tout à la volonté divine. On pressent les dangers d'une telle conception radicale de la perfection chrétienne qui soupçonne systématiquement une motivation égoïste derrière tout acte de dévotion, derrière chaque pratique vertueuse et chaque œuvre de charité. On peut y voir une radicalisation outrancière de la conception «extatique» de l'amour, qui d'après le P. Rousselot ${ }^{32}$ aurait vu le jour au $\mathrm{XII}^{\mathrm{e}}$ siècle sous la plume de certains mystiques dialecticiens tels Abélard ou Hugues de Saint-Victor, avant d'être intégrée à la scolastique des franciscains. Cette conception, ainsi

30. Voir le Primer Abecedario espiritual, édition de José Juan Morcillo Pérez, Madrid, Editorial Cisneros, 2004, prologue, p. 23.

31. Pour plus de détails et de précisions, nous nous permettons de renvoyer à notre thèse - spécialement le chapitre intitulé «La loi d'amour, une grande entreprise de réajustement doctrinal », p. 121-206.

32. Pierre Rousselot, Pour l'histoire du problème de l'amour au Moyen Âge (1908), Paris, Vrin, 1981, p. 3-4. 
dénommée parce qu'elle met le sujet aimant «hors de lui-même», présuppose une radicale hétérogénéité entre l'amour de Dieu et l'amour de soi: l'amour de Dieu sera d'autant plus «amour» et d'autant plus pur qu'il sera étranger à toute «inclination égoïste » ou naturelle. La perfection en amour requiert donc l'absolue gratuité, le plus total désintéressement.

Tout à l'encontre, Osuna se situe dans la ligne de la conception qualifiée par le P. Rousselot de «physique» - ou gréco-thomiste - de l'amour, qui établit une continuité, voire une identité foncière entre amour de Dieu et amour de soi, le premier émanant nécessairement de la «propension qu' ont tous les êtres de la nature à chercher leur propre bien». Il s'emploie, en s'appuyant sur Thomas d'Aquin qui relit les théories d'Aristote et du pseudo-Denys, à réconcilier amour de soi, amour du prochain et amour de Dieu. Il prend bien évidemment soin de distinguer très clairement l'amour de soi - auquel il consacre deux chapitres (30 et 31) au cœur de la Loi d'amour - de l'amour-propre et des intérêts égoïstes, de façon à réconcilier amour de soi et amour de Dieu, qui se retrouvent et coïncident dans la quête du Souverain Bien, vers où convergent tout naturellement les intérêts de l'homme et ceux de Dieu. Dans la perspective osunienne, s'aimer soi-même consiste à «aimer Dieu en soi-même ${ }^{33}$ », ce qui revient à s'aimer en Dieu. L'obligation d'amour de soi qui donne la mesure de l'amour du prochain dans le grand commandement évangélique (Marc, 12, 31) se décline sous la plume du franciscain en trois modalités, en fonction du degré de progression du chrétien: 1) s'aimer en Dieu, c'est d'abord se corriger, s'amender, et combattre ses inclinations égoïstes; 2) aimer Dieu en soi-même, c'est aussi rechercher la possession de Dieu pour soi, et orienter et subordonner précisément toutes ses actions à la quête de cette possession de Dieu; 3) enfin, s'aimer soi-même, c'est se livrer tout entier avec «véhémence» au pur amour de Dieu, car l'amour de soi est, en essence, amour de Dieu $^{34}$. L'amour de soi n'est donc par un obstacle au pur amour de Dieu; bien au contraire, il en est partie intégrante.

Avec l'amour de soi, Osuna réhabilite du même coup la possibilité de l'action vertueuse sans qu'il faille nécessairement y

33. Ley de amor, ch. 31, f. 127v: «creo que este amor de que Dios manda que te ames, más es amor suyo que tuyo, quiero decir que te manda que lo ames en ti ».

34. Ibid., ch. 31, f. 129v-130r: «que eches tu cuidado en el amor de Dios, porque amar a Dios es amar a ti mesmo». 
soupçonner un intérêt caché. En effet, l'homme, en aimant Dieu, travaille à son propre bien, de la même façon que, lorsqu'il fait le bien, il œuvre dans son intérêt tout en accomplissant la volonté de Dieu. La pratique des vertus est une des façons d'aimer Dieu, et il n'y a pas lieu de livrer un combat permanent contre toute action méritoire. Bien au contraire, Osuna rappelle que «la seule bonté morale ne suffit pas : la bonté méritoire est aussi nécessaire $»^{35}$.

L'amour du prochain ne représente pas non plus une entrave au pur amour de Dieu, comme semblaient le soutenir certains illuminés. Au contraire, écrit Osuna, «Dieu est un bien qui se multiplie lorsqu' on le donne; c'est pour cela que personne ne peut aimer parfaitement Dieu sans aimer parfaitement son prochain $»^{36}$.

Mieux encore, l'amour que le chrétien doit à son ennemi constitue un modèle d'amour «pur». En effet, l'amour que l'on porte par charité à notre ennemi, à l'encontre de nos inclinations naturelles, est nécessairement dénué de tout intérêt personnel, et constitue une forme d'amour «à l'état pur», libre de tout autre affect. La difficulté de l'amour en situation d'adversité permet de mettre à l'épreuve la pureté du sentiment amoureux. C'est ce qu'Osuna traduit par le biais de la métaphore du feu qui brûle en milieu humide:

Dans l'amour qu'au nom de Dieu nous portons à toutes les autres choses, l'on trouve toujours une part de notre amour qui gâche un peu l'amour de Dieu, tandis qu'aimer Dieu à travers notre ennemi garantit la pureté de cet amour [...] Le feu qui brûle en milieu humide est plus fort que celui qui brûle en milieu sec, car il y a une grande différence entre vaincre son ennemi et avoir la faveur de son ami. Lorsque ton amour vit et brûle en la personne de ton ennemi, sois assuré de la victoire du feu céleste et sacré qui t'anime, car l'humide inimitié n'est pas parvenue à l'éteindre ${ }^{37}$.

35. Ibid., ch. 32, f. 132r.

36. Ibid., ch. 48, f. 194r: «Porque el amor de Dios es de sí mesmo comunicativo, fallarás que cuanto uno más posee a Dios, más trabaja de lo dar a los otros, ca Dios es un bien que, dando, se multiplica, y por esto, ninguno puede ser perfecto en amar a Dios sin lo ser en amar al prójimo ».

37. Ibid., ch. 42, f. 170v: «En el amor que por Dios tenemos a todas las otras cosas fallamos algo de nuestro amor que agua un poco el amor de Dios, mas cuando ponemos el amor de Dios en nuestro enemigo, está muy puro y aun probado. (...) Más fuerte es el fuego que arde en la humedad que no el que arde en lo seco, porque gran diferencia hay de vencer al contrario a ser favorecido del amigo. Cuando tu amor vive y arde en tu enemigo, cree que tu fuego sancto y celestial se ha enseñoreado, pues la húmeda enemistad no lo ha podido apagar». 


\section{L'amour pur et la question des œuvres}

Nous venons de voir comment Osuna s'attache à travers la Loi d'amour, contre les excès des partisans de l'Abandon, à réhabiliter l'amour de soi et du prochain et, de façon générale, l'amour actif, qui émane de la volonté humaine. Il réaffirme à maintes reprises l'obligation pour le chrétien de pratiquer la charité à travers les bonnes œuvres. Il va même jusqu'à soutenir avec Bonaventure ${ }^{38}$ que l'amour est, par essence, œuvre et que, sans les œuvres, l'amour est condamné à demeurer un sentiment mort, car stérile, dont on est alors en droit de douter de l'authenticité. La position d'Osuna sur ce point n'est cependant pas dénuée d'ambiguïté. À certains endroits du traité, il semble en effet tenir les bonnes œuvres à distance et les reléguer à un second plan - inférieur - de la vie spirituelle, en soutenant par exemple au chapitre 33 que les œuvres extérieures n'augmentent pas la bonté de l'œuvre intérieure ${ }^{39}$. Au chapitre 48 , il va beaucoup plus loin en donnant subtilement à entendre qu'elles ne conduisent pas à l'amour parfait, voire même qu'elles font obstacle à sa puretét ${ }^{4}$.

La clé de ce paradoxe nous semble résider dans les différentes façons qu'a le franciscain d'interpréter la formule Amor est opus. Suivant une première acception, l'amour est «œuvre» au sens où il conduit tout naturellement à l'exécution d'œuvres de charité qui prolongent au-dehors le sentiment intérieur d'amour et lui donnent corps. Les œuvres sont le fruit naturel de l'amour et permettent de l'identifier comme tel. On devra donc en conclure qu'on ne peut concevoir d'amour sans œuvre extérieure, qui est en quelque sorte le baromètre de l'amour. Mais la formule est susceptible d'une seconde interprétation sensiblement différente, et qui conduit à la conclusion inverse : si l'amour est œuvre, c'est qu'il l'est par essence. Aimer, c'est déjà par définition œuvrer, sans qu'il y ait besoin

38. Ibid., ch. 33, f. 133v: «Pues, para que amemos como en el monte del amor divino nos es mostrado, habemos de notar que, según dice el seráfico doctor Sant Buenaventura, el amor de Dios más es efecto que afecto, más es obra que afeción», ce qui renvoie certainement à «quia dilectio Dei ad creaturam non est affectio, sed effectus communicatio» (Bonaventure, Commentaria in Quatuor Libros Sententiarum, Lib. I, d. 1, a. 3, q. 2, 3).

39. Ibid, ch. 33, f. 133v: «Dicen los teólogos que la obra esterior ninguna bondad añade a la interior, sino que en aquel bienquerer está toda la bondad de la obra y todo el amor».

40. Ibid., ch. 48, f. $192 \mathrm{v}$. 
d'ajouter à l'acte d'amour d'autres œuvres extérieures. L'amour est alors considéré comme la plus parfaite des œuvres, qui se suffit à elle-même, et qui mérite que l'on s'y consacre exclusivement. Les bonnes œuvres ne sont donc que de pâles valeurs ajoutées, dont peut se passer le pur amour, la plus sublime des œuvres.

Francisco de Osuna semble résoudre cette apparente contradiction en accordant aux bonnes œuvres une importance relative en fonction des différents niveaux de progression spirituelle ${ }^{41}$. Pour ceux qui débutent dans le cheminement spirituel, les bonnes œuvres occupent une place centrale, et constituent une sorte d'exercice qui tout à la fois prouve, fortifie et fait grandir l'amour de Dieu. Dans un deuxième temps, lorsque l'on avance sur le chemin de la perfection, la pratique des œuvres doit être orientée vers la seule gloire de l'amour de Dieu, sans que viennent se mêler d'autres motivations ou intérêts personnels. Il s'agira de pratiquer la charité par pur amour pour Dieu. Enfin, dans un troisième temps de l'itinéraire de l'amour de Dieu, auquel parviennent les seuls parfaits, la plénitude de l'amour est telle que toute œuvre autre que l'acte de pur amour lui-même est abandonnée. Les bonnes œuvres sont volontiers assimilées sous la plume d'Osuna à des «légumes embarrassants et peu nourrissants» (verdura que embaraz[a] y no $d[a]$ mantenimiento) pour les plus parfaits, que le seul pur amour vient rassasier ${ }^{42}$. De là qu'ils cherchent «souvent» (muchas veces) à se débarrasser de tout exercice et de toute œuvre extérieure pour «appliquer toute leur raison et leur attention à Dieu seul, sans aucun intermédiaire».

\section{Un amour pur, sans mélange et sans médiation:} la place de l'humanité du Christ

L'idée d'immédiateté est sous-jacente à toute quête de pur amour. Un amour pur, sans mélange, n'admet pas d'autre objet que la seule divinité. D'où les incessantes invitations, tout au long des pages de la Loi d'amour, à fixer son attention sur «Dieu seul», et à n'aimer que Dieu (amar a solo Dios). La quête intérieure et exclusive de pur amour constitue le cœur de la doctrine osunienne 
du Recueillement, que l'on peut définir comme une méthode d'oraison basée sur l'introspection, invitant à faire en soi le vide et à éliminer tout ce qui est extérieur, tout ce qui n'est pas Dieu. Un des principes fondamentaux du Recueillement est certainement le très révélateur no pensar nada («ne rien penser»), qu'Osuna défend vigoureusement au chapitre 26 , dans un plaidoyer efficace où il répond tour à tour aux objections de ses détracteurs, qui voudraient y voir une impiété scandaleuse, une forme de suspension et d'extase dangereuse où l'idée même de Dieu est annihilée. Tout en enracinant la doctrine du no pensar nada dans la théologie négative d'un Pseudo-Denys et les principes mystiques d'un Richard de SaintVictor, Osuna continue d'envisager le no pensar nada comme un véritable acte de foi: «ce rien penser, c'est penser tout», avait-il soutenu dans son Troisième Abécédaire ${ }^{43}$, et donc penser «le Tout». «Ne rien penser» signifie pour le franciscain évacuer de sa pensée tout ce qui n'est pas la pure divinité, ne plus se représenter que la simplicísima divinidad de Dios:

nous disons, ou plutôt, ceux qui parlent simplement disent, que ceux qui y pensent ne pensent rien, car eux-mêmes sont incapables de dire ce qu'ils pensent; et leur pensée n'est pas pensée de quelque chose que ce soit, mais une très simple représentation avec laquelle ils fixent très attentivement leur attention sur la très simple divinité de Dieu, sans l'en détourner vers quelque autre objet que ce soit ${ }^{44}$.

On entrevoit sans difficulté les délicates implications d'une telle conception : l'expérience d'un pur amour centré sur la seule divinité exclut logiquement toute idée d'humanité, fût-elle celle du Christ. Et de fait, précise Osuna, les artisans du Recueillement penseront à la «divinité du Christ» plutôt qu'à son humanitét5.

Il serait pourtant erroné de supposer chez notre franciscain un quelconque mépris pour l'humanité du Christ, lui qui consacre deux Abécédaires entiers - le premier et le sixième - à traiter de la méditation de la Passion et des plaies du Christ. Il ne manque pas

43. Tercera parte del Abecedario espiritual, tr. 21, ch. 5, p. 559.

44. Ley de amor, ch. 26, f. 108v: «decimos, o dicen los que hablan simplemente, que no piensan nada los que piensan en ello, porque ellos mesmos no pueden decir lo que piensan, ni esto que tienen es pensamiento de cosa alguna criada sino una muy sencilla representación con que afijan atentísimamente su intento en una simplicísima divinidad de Dios, sin variar de allí a cosa que sea».

45. Ibid., f. 109r-v: «la escondida divinidad de Cristo, en la cual piensan los varones recogidos sin la referir a otra cosa ninguna». 
non plus au chapitre 29 de la Loi d'amour d'expliquer comment il est possible de rechercher Dieu et de l'aimer à travers la méditation de la Passion. Ceci étant, il tient au nom du pur amour la considération de l'humanité du Sauveur à l'écart de l'expérience contemplative du Recueillement, qui n'a d'yeux que pour le divin. C'est là, d'après Emmanuel Renault ${ }^{46}$, ce qui fait principalement la différence entre le recueillement osunien et l'oraison de recueillement thérésienne, qui demeure quant à elle christocentrée.

\section{Les limites temporelles de l'expérience du pur amour}

Aimer Dieu d'un amour pur implique donc chez Osuna de «cesser de penser», de «cesser de prier», de «cesser d'œuvrer»: autant de principes constitutifs du Recueillement qui rappellent de façon troublante la suspension totale de toutes les facultés prêchées par les partisans de l'Abandon. L'enjeu est de taille au regard de l'institution ecclésiastique et de la société en général, car l'expérience mystique du pur amour de Dieu excluant a priori toute autre activité si noble soit-elle, il semble indispensable de baliser et de délimiter l'espace temporel de la contemplation, durant laquelle l'amoureux fervent de Dieu échappe à ses obligations courantes et se trouve pour ainsi dire soustrait aux codes qui régulent la vie en société, tout absorbé qu'il est par le pur amour de Dieu.

Sur la question de ces limites temporelles, le positionnement d'Osuna est tout en nuances. Il convient de rappeler tout d'abord les restrictions qui délimitent clairement dans la Loi d'amour le cadre de l'expérience mystique du pur amour: celle-ci ne survient qu'au neuvième et dernier degré de l'échelle de progression spirituelle, chez les plus parfaits amoureux de Dieu (los varones fervientes), et ne saurait en aucun cas être donnée au «commun» (el pueblo común). En outre, elle n'est jamais acquise une fois pour toutes, et le fait d'aimer Dieu d'un amour pur et parfait - autant que faire se peut ici-bas - n'implique pas pour le franciscain de l'aimer «continûment», sans interruption. Il considère en effet l'amour continu comme une fiction: la nature de l'homme étant par définition imparfaite et inconstante, il lui est impossible de

46. Emmanuel Renault, L'oraison thérésienne (1999), Toulouse, Éditions du Carmel, 2010, p. 24-26. 
prolonger indéfiniment l'état contemplatif au point de prodiguer sans discontinuer à Dieu un amour absolu. Et quand bien même il en serait capable, le pur amour continu ne semble ni souhaitable ni compatible avec les obligations de la piété et de la vie courante dont nul n'est exempt:

Nous quittons donc souvent l'état d'amour actuel, et nous le laissons de côté pour aider Marthe; et pour devenir Marthe, il est nécessaire que Marie se lève et qu'elle abandonne son repos pour que d'autres puissent se reposer, et qu'elle-même s'exerce aux œuvres de miséricorde, auxquelles nous sommes tout autant obligés qu'à l'amour de Dieu; et comme il est impossible à l'homme de prêter attention à plusieurs choses à la fois, l'acte d'amour de Dieu faiblit momentanément, voire même cesse complètement, sans que disparaissent pour autant ni l'habitude ni le désir de l'aimer à nouveau et de retrouver la quiétude première, sans trêve ${ }^{47}$.

La clé du raisonnement osunien repose ici sur la distinction entre les concepts d'«amour actuel» (amor actual) et d' «amour virtuel» (amor virtual). Le pur amour actuel de Dieu peut parfois être interrompu, sans que cesse pour autant l'amour virtuel. Cela étant bien établi, quelle peut être la durée maximale de l'expérience du pur amour? Au chapitre 27 de la Loi d'amour, Osuna limite à une «demi-heure» le temps réel qui est susceptible d'être consacré exclusivement à l'expérience mystique du Recueillement, qui n'a d'autre contenu que le pur amour de Dieu. On constate que cette durée a été revue à la baisse depuis la publication du Troisième Abécédaire spirituel $^{48}$ en 1527 , qui prévoyait la possibilité de consacrer une à deux heures par jour au Recueillement. On peut sans doute y voir un prudent compromis du franciscain avec les exigences et les critères de l'orthodoxie, en ces temps de polémiques et de procès.

Il reste que Francisco de Osuna encourage l'âme de son lecteur à «continuer» le plus possible l'exercice du Recueillement, à cultiver

47. Ley de amor, ch. 22, f. 90v: «Levantámonos, pues, muchas veces del actual amor, y dejámoslo por ayudar a Marta; y aun por se hacer Marta es menester que se levante María y deje su reposo por dar a otros reposo, y ejercitarse en las obras de misericordia, a que también somos obligados como al amor de Dios; y como sea imposible estar el hombre juntamente atento a muchas cosas, afloja por entonces y aun del todo cesa el acto del amor de Dios, aunque no el hábito ni el deseo de lo tornar a amar con el reposo primero, sin cesar».

48. Tercer Abecedario espiritual, tr. 15, ch. 2, édition citée (supra note 2) p. 399. 
le désir d'amour divin, à s'efforcer de soutenir et d'appliquer son attention à Dieu seul. Tout en étant conscient qu'il s'agit d'un phénomène sporadique, il est partisan de préserver un «espace», entendu comme moment, qui soit dédié uniquement, et donc à l'exclusion de tout le reste, au pur amour de Dieu: «Apprends, mon âme, à aimer ton Dieu ne serait-ce qu'un court instant sans faire pour lors aucune autre chose $»^{49}$.

S'il écarte l'éventualité de l'amour continu - concept qui sera approfondi par Miguel de Molinos aux chapitres 14 et 15 du livre I du Guide spirituel -, Osuna ne perd pas de vue la possibilité de goûter au pur amour «ne serait-ce qu'un court instant»; il ne renonce donc pas à ce qui fait la substantifique moelle de son enseignement mystique du Recueillement: aimer Dieu d'un amour pur, sans mélange ni médiation, et vaquer à Dieu seul, vacar a sólo Dios.

Ainsi, Francisco de Osuna réserve à l'expérience mystique du pur amour une place de choix dans son Quatrième Abécédaire, au point d'en faire la clef de voûte de tout l'édifice spirituel de la Loi d'amour. Son enseignement n'atteint cependant pas le degré de systématicité de la doctrine fénelonienne du pur amour. Le franciscain andalou procède plutôt par tâtonnements, par touches discrètes, et se voit contraint à un certain nombre de réajustements doctrinaux successifs afin de répondre au mieux aux problèmes théologiques soulevés lors de la condamnation des thèses de l'Abandon, dans un contexte de forte pression inquisitoriale. Il consacre donc toute son énergie à trouver une formulation plus nuancée et moins intransigeante de la doctrine du pur amour, afin de la rendre acceptable au regard d'une orthodoxie de plus en plus sur le qui-vive. Pour ce faire, il replace tout naturellement la quête du pur amour au cœur de la vie spirituelle: il le présente comme le plein accomplissement du grand commandement d'amour, l'ultime échelon de la perfection vers lequel tout chrétien doit tendre. Il ne cède cependant pas sur ce qui fait la spécificité de l'enseignement mystique du Recueillement, en ménageant au pur amour un espace, si réduit soit-il, qui échappe aux codes habituels

49. Ley de amor, ch. 48, f. 192v: «Aprende, ánima mía, a amar algún pequeño espacio siquiera a tu Dios sin hacer por entonces otra cosa alguna». 
de la piété traditionnelle: au nom du pur amour, les prières vocales, les œuvres de charité et la considération de l'humanité du Christ sont momentanément tenues à l'écart de la plus haute expérience de l'amour divin, ce qui constitue un positionnement audacieux en ces temps de lutte contre l'illuminisme. Il n'y a pas toutefois chez Osuna, comme c'est le cas déjà chez Henri Harphius ${ }^{50}$ ou comme plus tard chez Fénelon, d'exigence de parfait désintéressement. Le pur amour osunien, passé au tamis de la censure, a été contraint de trouver une formulation de compromis. Il en ressort un pur amour moins radical, et certainement moins exigeant moralement, mais qui n'a pas perdu pour autant de son feu ni de son éclat.

estelle.garbay@casadevelazquez.org

estelle.garbay@yahoo.fr

2, ruelle de la Fontaine

21220 Gemeaux

50. On trouve en effet déjà chez Harphius l'idée selon laquelle le pur amour de Dieu implique chez celui qui en fait l'expérience une absolue indifférence aussi bien à la promesse de la Gloire qu'aux possibles souffrances de l'Enfer, si tel était le dessein de Dieu. Voir Harph (Henricus), Theologia mystica cum speculativa, tum praecipue affectiva, Cologne, Melchior Novesian, 1538, Liber III, sermo XIII («De eadem industria qualitater exercetur iri coelica sanitate et infernali languore»), f. 206r: «Moriens ergo sibi, divino beneplacito totaliter se offerat, dicens: Fiat voluntas tua, tam in adversis quam in prosperis. Totus enim tuus sum domine, tolle quod tuum est et vade: quia paratum me senties ire, sive in infernum, sive in paradisum, si tuae dulcedini sic complacuerit». Consultable en ligne [http:// reader.digitale-sammlungen.de/de/fs1/object/display/bsb10148816_00001.html]. 